\title{
Solvability of an Integral Equation of Volterra-Wiener-Hopf Type
}

\author{
Nurgali K. Ashirbayev, ${ }^{1}$ Józef Banas,, ${ }^{2}$ and Agnieszka Dubiel ${ }^{2}$ \\ ${ }^{1}$ Department of Mathematics, Science-Pedagogical Faculty, M. Auezov South Kazakhstan State University, Tauke Khan Avenue 5, \\ Shymkent 160012, Kazakhstan \\ ${ }^{2}$ Department of Mathematics, Rzeszów University of Technology, Al. Powstanców Warszawy 8, 35-359 Rzeszów, Poland
}

Correspondence should be addressed to Józef Banaś; jbanas@prz.edu.pl

Received 10 March 2014; Accepted 1 May 2014; Published 13 May 2014

Academic Editor: Xiao-Jun Yang

Copyright (C) 2014 Nurgali K. Ashirbayev et al. This is an open access article distributed under the Creative Commons Attribution License, which permits unrestricted use, distribution, and reproduction in any medium, provided the original work is properly cited.

The paper presents results concerning the solvability of a nonlinear integral equation of Volterra-Stieltjes type. We show that under some assumptions that equation has a continuous and bounded solution defined on the interval $[0, \infty)$ and having a finite limit at infinity. As a special case of the mentioned integral equation we obtain an integral equation of Volterra-Wiener-Hopf type. That fact enables us to formulate convenient and handy conditions ensuring the solvability of the equation in question in the class of functions defined and continuous on the interval $[0, \infty)$ and having finite limits at infinity.

\section{Introduction}

Integral equations play very important and significant role in the description of numerous events appearing in real world. Almost all branches of physics, mathematical physics, engineering, astronomy, economics, biology, and so forth utilize the theory of integral equations, both linear and nonlinear (cf. [1-5], e.g.).

Integral equations of Wiener-Hopf type create very important branch of the theory of integral equations [5]. Integral equations of such a type belong to the part of the theory of integral equations which are often called as integral equations depending on the difference of arguments [5]. It is worthwhile mentioning that integral equations of WienerHopf type find numerous applications. For example, they are applied to describe some problems of radiative equilibria [6] and in the theory of diffraction [7]. Moreover, the reflection of an electromagnetic plane wave by an infinite sets of plates is also investigated with help of Wiener-Hopf integral equations [8]. Other possible applications of the theory of Wiener-Hopf integral equations are associated with dynamic elasticity [9], diffraction of plane waves by circular cone [10], and so forth (cf. also [5]).

Let us recall that the classical Wiener-Hopf integral equation has the form

$$
x(t)=a(t)+\int_{a}^{b} k(t-s) f(s, x(s)) d s,
$$

where $t \in[a, b]$ and $k: \mathbb{R} \rightarrow \mathbb{R}$ is a given function which is continuous and integrable on the set of real numbers $\mathbb{R}$; that is, there exists a finite improper integral:

$$
\int_{-\infty}^{+\infty} k(u) d u
$$

Obviously, instead of (1) we may consider its "unbounded domain" counterpart having the form

$$
x(t)=a(t)+\int_{0}^{\infty} k(t-s) f(s, x(s)) d s
$$

or even more general equations [5]. 
In this paper we will investigate the Volterra counterpart of the Wiener-Hopf integral equations (1) and (3), which has the form

$$
x(t)=a(t)+\int_{0}^{t} k(t-s) f(s, x(s)) d s,
$$

where $t \in \mathbb{R}_{+}$or $t \in[0, T]$ with $T>0$.

Let us pay attention to the fact that Volterra-Wiener-Hopf integral equation (4) appears quite naturally as a special case of (1) and (3). In fact, if we require that

$$
k(u)=0 \quad \text { for } u \leqslant 0,
$$

then (3) reduces to (4). This observation justifies the interest in the study of the Volterra-Wiener-Hopf integral equations.

To make our investigations more general and more convenient, we will study the so-called Volterra-Stieltjes integral equation having the form

$$
x(t)=a(t)+\int_{0}^{t} f(s, x(s)) d_{s} K(t, s),
$$

where the involved integral is understood in the RiemannStieltjes sense.

The details explaining such an approach as well as suitable definitions will be presented in our further considerations.

\section{Notation, Definitions, and Auxiliary Facts}

In this sectio, $\mathrm{n}$ we present notation, definitions, and all auxiliary facts which will be utilized further on. Similar to Section 1 , we will denote by $\mathbb{R}$ the set of real numbers. We put also $\mathbb{R}_{+}=[0, \infty)$.

The investigation of the paper will be conducted in the Banach function space $\mathrm{BC}\left(\mathbb{R}_{+}\right)$consisting of all real functions defined, continuous, and bounded on the interval $\mathbb{R}_{+}$. This space is endowed by the classical supremum norm

$$
\|x\|=\sup \{|x(t)|: t \geqslant 0\} .
$$

Let us notice that in the space $\mathrm{BC}\left(\mathbb{R}_{+}\right)$the classical Ascoli-Arzela criterion for relative compactness fails to work and we know only a few sufficient conditions guaranteeing the relative compactness (cf. $[11,12])$. Keeping in mind our further purposes we provide below a sufficient condition of such a type [12].

Theorem 1. Let $X$ be a nonempty and bounded subset of the space $B C\left(\mathbb{R}_{+}\right)$. Assume that $X$ is locally equicontinuous; that is, for any $T>0$, the functions from $X$ are equicontinuous on the interval $[0, T]$. Moreover assume that the following condition is satisfied.

For any $\varepsilon>0$ there exists a number $T>0$ such that for any function $x \in X$ and for all $t, s \in[T, \infty)$ the inequality $|x(t)-x(s)| \leqslant \varepsilon$ is satisfied.

Then the set $X$ is relatively compact in the space $B C\left(\mathbb{R}_{+}\right)$.

Remark 2. Let us notice that in the case when a set $X$ satisfies conditions imposed in Theorem 1 all functions from $X$ tend to finite limits at infinity uniformly with respect to the set $X$ (cf. $[11,12])$.

In the sequel we will use the concept of the modulus of continuity of a function from the space $\mathrm{BC}\left(\mathbb{R}_{+}\right)$. Thus, fix arbitrarily $T>0$ and take a function $x \in \mathrm{BC}\left(\mathbb{R}_{+}\right)$.

Consider the quantity

$$
\omega^{T}(x, \varepsilon)=\sup \{|x(t)-x(s)|: t, s \in[0, T],|t-s| \leqslant \varepsilon\}
$$

defined for $\varepsilon>0$. This quantity is called the modulus of continuity of a function $x$ on the interval $[0, T]$. Obviously $\lim _{\varepsilon \rightarrow 0} \omega^{T}(x, \varepsilon)=0$ in view of the uniform continuity of $x$ on the interval $[0, T]$.

Now, we provide needed facts concerning functions of bounded variation [13].

At the beginning assume that $x$ is a real function defined on a fixed interval $[a, b]$. Then the symbol $\bigvee_{a}^{b} x$ will denote the variation of the function $x$ on the interval $[a, b]$. In the case when $\bigvee_{a}^{b} x$ is finite we say that $x$ is of bounded variation on $[a, b]$. If we have a function $u(t, s)=u:[a, b] \times[c, d] \rightarrow \mathbb{R}$, then we denote by $\bigvee_{t=p}^{q} u(t, s)$ the variation of the function $t \rightarrow u(t, s)$ on the interval $[p, q] \subset[a, b]$, where $s$ is a fixed number in the interval $[c, d]$. Similarly we define the quantity $\bigvee_{s=p}^{q} u(t, s)$.

For the properties of functions of bounded variation we refer to [13].

If $x$ and $\varphi$ are two real functions defined on the interval $[a, b]$ then under some additional conditions [13] we can define the Stieltjes integral (in the Riemann-Stieltjes sense)

$$
\int_{a}^{b} x(t) d \varphi(t)
$$

of the function $x$ with respect to the function $\varphi$. In such a case we say that $x$ is Stieltjes integrable on the interval $[a, b]$ with respect to $\varphi$.

Let us mention that several conditions are known which guarantee the Stieltjes integrability $[3,13,14]$. One of the most frequently used conditions requires that $x$ is continuous and $\varphi$ is of bounded variation on $[a, b]$.

In what follows we will utilize a few properties of the Stieltjes integral contained in the following given lemmas [13].

Lemma 3. If $x$ is Stieltjes integrable on the interval $[a, b]$ with respect to a function $\varphi$ of bounded variation, then

$$
\left|\int_{a}^{b} x(t) d \varphi(t)\right| \leqslant \int_{a}^{b}|x(t)| d\left(\bigvee_{a}^{t} \varphi\right) .
$$

Lemma 4. Let $x_{1}$ and $x_{2}$ be Stieltjes integrable functions on the interval $[a, b]$ with respect to a nondecreasing function $\varphi$ such that $x_{1}(t) \leqslant x_{2}(t)$ for $t \in[a, b]$. Then

$$
\int_{a}^{b} x_{1}(t) d \varphi(t) \leqslant \int_{a}^{b} x_{2}(t) d \varphi(t) .
$$

Further on, we will also consider Stieltjes integrals having the form

$$
\int_{a}^{b} x(s) d_{s} g(t, s)
$$


where $g:[a, b] \times[a, b] \rightarrow \mathbb{R}$ and the symbol $d_{s}$ indicates the integration with respect to $s$. The details concerning the integral of this type will be given later. Let us only mention that integral (12) allows us to represent the Volterra-WienerHopf integral equation (4) as a particular case of the VolterraStieltjes integral equation (6).

\section{Main Results}

The investigations of this section will be located in the Banach function space $\mathrm{BC}\left(\mathbb{R}_{+}\right)$described previously in Section 2 . Firstly, we will consider the solvability of the Volterra-Stieltjes integral equation having form (6). This equation will be studied under the following formulated assumptions.

(i) The function $a=a(t)$ belongs to the space $\mathrm{BC}\left(\mathbb{R}_{+}\right)$ and is such that there exists the $\operatorname{limit}_{t \rightarrow \infty} a(t)$ (obviously, this limit is finite).

(ii) $f: \mathbb{R}_{+} \times \mathbb{R} \rightarrow \mathbb{R}$ is continuous and there exists a function $\phi: \mathbb{R}_{+} \rightarrow \mathbb{R}_{+}$which is nondecreasing, $\phi(0)=0, \lim _{t \rightarrow 0} \phi(t)=0$, and such that

$$
|f(t, x)-f(t, y)| \leqslant \phi(|x-y|)
$$

for all $t \in \mathbb{R}_{+}$and $x, y \in \mathbb{R}$.

(iii) The function $t \rightarrow f(t, 0)$ is a member of $\mathrm{BC}\left(\mathbb{R}_{+}\right)$.

(iv) $K(t, s)=K: \Delta \rightarrow \mathbb{R}$ is a uniformly continuous function on the triangle

$$
\Delta=\{(t, s): 0 \leqslant s \leqslant t\} .
$$

(v) The function $s \rightarrow K(t, s)$ is of bounded variation on the interval $[0, t]$ for each fixed $t \in \mathbb{R}_{+}$.

(vi) For any $\varepsilon>0$ there exists $\delta>0$ such that for all $t_{1}, t_{2} \in$ $\mathbb{R}_{+}$with $t_{1}<t_{2}, t_{2}-t_{1} \leqslant \delta$, the following inequality holds:

$$
\bigvee_{s=0}^{t_{1}}\left[K\left(t_{2}, s\right)-K\left(t_{1}, s\right)\right] \leqslant \varepsilon .
$$

(vii) $K(t, 0)=0$ for all $t \geqslant 0$.

(viii) The function $t \rightarrow \bigvee_{s=0}^{t} K(t, s)$ is bounded on $\mathbb{R}_{+}$.

Before formulating the last assumption let us denote by $F_{1}$ and $\bar{K}$ the following constants:

$$
\begin{gathered}
F_{1}=\sup \left\{|f(t, 0)|: t \in \mathbb{R}_{+}\right\}, \\
\bar{K}=\sup \left\{\bigvee_{s=0}^{t} K(t, s): t \in \mathbb{R}_{+}\right\} .
\end{gathered}
$$

Obviously $F_{1}<\infty$ in view of assumption (iii), while the inequality $\bar{K}<\infty$ is a consequence of assumption (viii).

Now, we can formulate our last assumption.

(ix) There exists a positive solution $r_{0}$ of the inequality

$$
\|a\|+\left(\phi(r)+F_{1}\right) \bar{K} \leqslant r .
$$

Now, we are prepared to present our first main result.
Theorem 5. Under assumptions (i)-(ix), (6) has at least one solution $x=x(t)$ in the space $B C\left(\mathbb{R}_{+}\right)$which belongs to the ball $B_{r_{0}}=\left\{x \in B C\left(\mathbb{R}_{+}\right):\|x\| \leqslant r_{0}\right\}$ and has a finite limit at infinity.

In the proof of the above theorem we will need a few auxiliary facts contained in the following given lemmas.

Lemma 6. The function

$$
p \longrightarrow \bigvee_{s=0}^{p} K(t, s)
$$

is continuous on the interval $[0, t]$ for any fixed $t \in \mathbb{R}_{+}$.

This lemma is an easy consequence of assumptions (iv) and (v) and the properties of the variation of functions (cf. [13], p. 60).

Lemma 7. Let assumptions (iv)-(vi) be satisfied. Then, for arbitrarily fixed number $t_{2}>0$ and for any $\varepsilon>0$, there exists $\delta>0$ such that if $t_{1}<t_{2}$ and $t_{2}-t_{1} \leqslant \delta$ then

$$
\bigvee_{s=t_{1}}^{t_{2}} K\left(t_{2}, s\right) \leqslant \varepsilon
$$

Proof. Fix $t_{2} \in(0, \infty)$ and $\varepsilon>0$. Next, consider the function $\mathrm{H}$ defined on the interval $\left[0, t_{2}\right]$ by the formula

$$
H(p)=\bigvee_{s=0}^{p} K\left(t_{2}, s\right) .
$$

Then, in view of Lemma 6 , the function $H$ is continuous at the point $t_{2}$. Hence we infer that there exists $\delta>0$ such that for $t_{1} \geqslant 0, t_{1}<t_{2}$, and $t_{2}-t_{1} \leqslant \delta$ we have that $\left|H\left(t_{2}\right)-H\left(t_{1}\right)\right| \leqslant$ $\varepsilon$. On the other hand, we get

$$
\begin{aligned}
\left|H\left(t_{2}\right)-H\left(t_{1}\right)\right| & =\left|\bigvee_{s=0}^{t_{2}} K\left(t_{2}, s\right)-\bigvee_{s=0}^{t_{1}} K\left(t_{2}, s\right)\right| \\
& =\left|\bigvee_{s=0}^{t_{1}} K\left(t_{2}, s\right)+\bigvee_{s=t_{1}}^{t_{2}} K\left(t_{2}, s\right)-\bigvee_{s=0}^{t_{1}} K\left(t_{2}, s\right)\right| \\
& =\bigvee_{s=t_{1}}^{t_{2}} K\left(t_{2}, s\right) \leqslant \varepsilon .
\end{aligned}
$$

The proof is complete.

Proof of Theorem 5. Let us consider the operator $F$ defined on the space $\mathrm{BC}\left(\mathbb{R}_{+}\right)$in the following way:

$$
(F x)(t)=a(t)+\int_{0}^{t} f(s, x(s)) d_{s} K(t, s)
$$

for $x \in \mathrm{BC}\left(\mathbb{R}_{+}\right)$and for arbitrarily fixed $t \in \mathbb{R}_{+}$. Then, keeping in mind the imposed assumptions, we deduce that the function $F x$ is well defined. 
Further, fix arbitrarily $T>0$ and take $s, t \in[0, T]$. Without loss of generality we may assume that $s<t$. Then, in view of Lemmas 3 and 4, we obtain

$$
\begin{aligned}
& |(F x)(t)-(F x)(s)| \\
& \leqslant|a(t)-a(s)| \\
& +\mid \int_{0}^{t} f(\tau, x(\tau)) d_{\tau} K(t, \tau) \\
& -\int_{0}^{s} f(\tau, x(\tau)) d_{\tau} K(s, \tau) \\
& \leqslant \omega^{T}(a, \varepsilon)+\mid \int_{0}^{t} f(\tau, x(\tau)) d_{\tau} K(t, \tau) \\
& -\int_{0}^{s} f(\tau, x(\tau)) d_{\tau} K(t, \tau) \\
& +\mid \int_{0}^{s} f(\tau, x(\tau)) d_{\tau} K(t, \tau) \\
& -\int_{0}^{s} f(\tau, x(\tau)) d_{\tau} K(s, \tau) \\
& \leqslant \omega^{T}(a, \varepsilon)+\left|\int_{s}^{t} f(\tau, x(\tau)) d_{\tau} K(t, \tau)\right| \\
& +\left|\int_{0}^{s} f(\tau, x(\tau)) d_{\tau}[K(t, \tau)-K(s, \tau)]\right| \\
& \leqslant \omega^{T}(a, \varepsilon) \\
& +\int_{s}^{t}|f(\tau, x(\tau))| d_{\tau}\left(\bigvee_{p=0}^{\tau} K(t, p)\right) \\
& +\int_{0}^{s}|f(\tau, x(\tau))| d_{\tau} \\
& \times\left(\bigvee_{q=0}^{\tau}[K(t, q)-K(s, q)]\right) \\
& \leqslant \omega^{T}(a, \varepsilon) \\
& +\int_{s}^{t}[|f(\tau, x(\tau)) .-f(\tau, 0)| \\
& +|f(\tau, 0)|] d_{\tau}\left(\bigvee_{p=0}^{\tau} K(t, p)\right) \\
& +\int_{0}^{s}[|f(\tau, x(\tau)) .-f(\tau, 0)| \\
& +|f(\tau, 0)|] d_{\tau}\left(\bigvee_{q=0}^{\tau}[K(t, q)-K(s, q)]\right) \\
& \leqslant \omega^{T}(a, \varepsilon)+\int_{s}^{t}\left\{\phi(|x(\tau)|)+F_{1}\right\} d_{\tau} \\
& \times\left(\bigvee_{p=0}^{\tau} K(t, p)\right)
\end{aligned}
$$

$$
\begin{aligned}
& +\int_{0}^{s}\left\{\phi(|x(\tau)|)+F_{1}\right\} d_{\tau} \\
& \times\left(\bigvee_{q=0}^{\tau}[K(t, q)-K(s, q)]\right) \\
\leqslant & \omega^{T}(a, \varepsilon)+\left\{\phi(\|x\|)+F_{1}\right\} \\
& \times \int_{s}^{t} d_{\tau}\left(\bigvee_{p=0}^{\tau} K(t, p)\right) \\
& +\left\{\phi(\|x\|)+F_{1}\right\} \\
& \times \int_{0}^{s} d_{\tau}\left(\bigvee_{q=0}^{\tau}[K(t, q)-K(s, q)]\right) \\
\leqslant & \omega^{T}(a, \varepsilon)+\left\{\phi(\|x\|)+F_{1}\right\} \bigvee_{p=s}^{t} K(t, p) \\
& +\left\{\phi(\|x\|)+F_{1}\right\} \bigvee_{q=0}^{s}[K(t, q)-K(s, q)] .
\end{aligned}
$$

Hence, in view of assumption (vi) and Lemma 7, we conclude that the function $F x$ is continuous on the interval $[0, T]$. Since $T$ was chosen arbitrarily this allows us to infer that $F x$ is continuous on $\mathbb{R}_{+}$.

Next, we show that the function $F x$ is bounded on $\mathbb{R}_{+}$. To this end, fix arbitrarily $x \in \mathrm{BC}\left(\mathbb{R}_{+}\right)$and $t \geqslant 0$. Then, in virtue of the imposed assumptions and Lemmas 3 and 4, we get

$$
\begin{aligned}
|(F x)(t)| \leqslant & \|a\|+\int_{0}^{t}|f(s, x(s))| d_{s}\left(\bigvee_{p=0}^{s} K(t, p)\right) \\
\leqslant & \|a\|+\int_{0}^{t}[|f(s, x(s))-f(s, 0)|+|f(s, 0)|] d_{s} \\
& \times\left(\bigvee_{p=0}^{s} K(t, p)\right) \\
\leqslant & \|a\|+\int_{0}^{t}\left[\phi(|x(s)|)+F_{1}\right] d_{s}\left(\bigvee_{p=0}^{s} K(t, p)\right) \\
\leqslant & \|a\|+\left\{\phi(\|x\|)+F_{1}\right\} \int_{0}^{t} d_{s}\left(\bigvee_{p=0}^{s} K(t, p)\right) \\
\leqslant & \|a\|+\left\{F_{1}+\phi(\|x\|)\right\} \bigvee_{s=0}^{t} K(t, s) .
\end{aligned}
$$

Now, in view of assumption (viii), we conclude that the following inequality holds:

$$
\|F x\| \leqslant\|a\|+\left\{F_{1}+\phi(\|x\|)\right\} \bar{K} .
$$

The above inequality shows that the function $F x$ is bounded on $\mathbb{R}_{+}$. This fact in connection with the continuity of 
the function $F x$ established above shows that $F x \in \mathrm{BC}\left(\mathbb{R}_{+}\right)$. In other words, the operator $F$ is a self-mapping of the space $\mathrm{BC}\left(\mathbb{R}_{+}\right)$.

Moreover, on the basis of inequality (25) and assumption (ix), we conclude that there exists a positive number $r_{0}$ such that the operator $F$ transforms the ball $B_{r_{0}}$ (see assumption (ix)) into itself.

Now we show that the operator $F$ is continuous on the ball $B_{r_{0}}$. To this end, fix $\varepsilon>0$. Next, fix arbitrarily $x, y \in B_{r_{0}}$ such that $\|x-y\| \leqslant \varepsilon$. Then, taking into account the imposed assumptions, for an arbitrary fixed number $t \in \mathbb{R}_{+}$, we get

$$
\begin{aligned}
& |(F x)(t)-(F y)(t)| \\
& \quad=\left|\int_{0}^{t}[f(s, x(s))-f(s, y(s))] d_{s} K(t, s)\right| \\
& \quad \leqslant \int_{0}^{t}|f(s, x(s)) .-f(s, y(s))| d_{s}\left(\bigvee_{p=0}^{s} K(t, p)\right) \\
& \quad \leqslant \int_{0}^{t} \phi(|x(s)-y(s)|) d_{s}\left(\bigvee_{p=0}^{s} K(t, p)\right) \\
& \quad \leqslant \int_{0}^{t} \phi(\varepsilon) d_{s}\left(\bigvee_{p=0}^{s} K(t, p)\right) \\
& \quad \leqslant \phi(\varepsilon) \bigvee_{s=0}^{t} K(t, s) \leqslant \bar{K} \phi(\varepsilon) .
\end{aligned}
$$

The above obtained estimate (26) shows that the operator $F$ is continuous even on the whole space $\mathrm{BC}\left(\mathbb{R}_{+}\right)$.

Now we show that the set $F\left(B_{r_{0}}\right)$ is relatively compact in the space $\mathrm{BC}\left(\mathbb{R}_{+}\right)$. To show this fact we introduce two auxiliary functions $M=M(\varepsilon)$ and $N=N(\varepsilon)$ defined in the following way:

$$
\begin{gathered}
M(\varepsilon)=\sup \left\{\bigvee_{s=0}^{t_{1}}\left[K\left(t_{2}, s\right)-K\left(t_{1}, s\right)\right]: t_{1}, t_{2} \in \mathbb{R}_{+},\right. \\
\left.t_{1}<t_{2}, t_{2}-t_{1} \leqslant \varepsilon\right\}, \\
N(\varepsilon)=\sup \left\{\bigvee_{s=t_{1}}^{t_{2}} K\left(t_{2}, s\right): t_{1}, t_{2} \in \mathbb{R}_{+}, t_{1}<t_{2}, t_{2}-t_{1} \leqslant \varepsilon\right\} .
\end{gathered}
$$

Observe that in view of assumption (iv) and Lemma 7 we have that $M(\varepsilon) \rightarrow 0$ and $N(\varepsilon) \rightarrow 0$ as $\varepsilon \rightarrow 0$.

Further, fix arbitrarily $\varepsilon>0$ and $T>0$ and choose a function $x \in B_{r_{0}}$. Next, take $t, s \in[0, T]$ such that $|t-s| \leqslant \varepsilon$. Without loss of generality we may assume that $s<t$. Then, in view of estimate (23), we get

$$
\begin{gathered}
|(F x)(t)-(F x)(s)| \leqslant \omega^{T}(a, \varepsilon)+\left[\phi\left(r_{0}\right)+F_{1}\right] N(\varepsilon) \\
+\left[\phi\left(r_{0}\right)+F_{1}\right] M(\varepsilon) .
\end{gathered}
$$

This estimate shows that functions from the set $F\left(B_{r_{0}}\right)$ are equicontinuous on the interval $[0, T]$.
Next, taking arbitrarily $t, s \in[T, \infty)$ with $s<t$ and arguing in the same way as we done in order to obtain estimate (23), we get

$$
\begin{aligned}
|(F x)(t)-(F x)(s)| \leqslant & |a(t)-a(s)| \\
& +\left\{\phi\left(r_{0}\right)+F_{1}\right\}[M(\varepsilon)+N(\varepsilon)] .
\end{aligned}
$$

Hence, keeping in mind assumption (i), we can choose $T>0$ so big that the term $|a(t)-a(s)|$ is suitably small for $s, t>T$. This assertion in conjunction with the fact that $M(\varepsilon) \rightarrow 0$ and $N(\varepsilon) \rightarrow 0$ as $\varepsilon \rightarrow 0$, in view of Theorem 1 , allows us to deduce that the set $F\left(B_{r_{0}}\right)$ is relatively compact in the space $\mathrm{BC}\left(\mathbb{R}_{+}\right)$.

Now, taking into account the continuity of the operator $F$ and applying the classical Schauder fixed point principle, we conclude that there exists at least one fixed point $x$ of the operator $F$ which belongs to the ball $B_{r_{0}}$. Obviously, the function $x=x(t)$ is a solution of the Volterra-Stieltjes integral equation (6). Moreover, let us notice that any fixed point $x=x(t)$ of the operator $F$ from the ball $B_{r_{0}}$ must belong to the set $F\left(B_{r_{0}}\right)$ being relatively compact in the sense of Theorem 1 . In the light of Remark 2 this fact allows us to infer that the function $x=x(t)$ being a solution of (6) has a finite limit at infinity.

The proof is complete.

Now, we pay our attention to assumption (vi) playing a key role in our investigations. It turns out that we can formulate a condition being handy in applications and ensuring that the function $K=K(t, s)$ satisfies assumption (vi).

To formulate that condition assume, as previously, that $K(t, s)=K: \Delta \rightarrow \mathbb{R}$, where $\Delta=\{(t, s): 0 \leqslant s \leqslant t\}$. Then, the announced condition may be formulated as follows.

$\left(\mathrm{vi}^{\prime}\right)$ For arbitrary $t_{1}, t_{2} \in \mathbb{R}_{+}$such that $t_{1}<t_{2}$ the function $s \rightarrow K\left(t_{2}, s\right)-K\left(t_{1}, s\right)$ is nonincreasing on the interval $\left[0, t_{1}\right]$.

Remark 8. The above condition and its consequences were discussed in [15] (cf. also [16]) under the assumption that $K$ : $\Delta_{1} \rightarrow \mathbb{R}$, where $\Delta_{1}=\{(t, s): 0 \leqslant s \leqslant t \leqslant 1\}$. Moreover, instead of $t_{1}, t_{2} \in \mathbb{R}_{+}$it was assumed that $t_{1}, t_{2} \in[0,1]$.

Further, we prove a few consequences of condition $\left(\mathrm{vi}^{\prime}\right)$.

Lemma 9. Under assumptions ( $\left.v i^{\prime}\right)$ and (vii), for arbitrarily fixed $s \in \mathbb{R}_{+}$, the function $t \rightarrow K(t, s)$ is nonincreasing on the interval $[s, \infty)$.

Proof. Fix a number $s \in \mathbb{R}_{+}$and take arbitrarily $t_{1}, t_{2} \in[s, \infty)$ with $t_{1}<t_{2}$. Then, in virtue of $\left(\mathrm{vi}^{\prime}\right)$, we obtain

$$
K\left(t_{2}, s\right)-K\left(t_{1}, s\right) \leqslant K\left(t_{2}, 0\right)-K\left(t_{1}, 0\right) .
$$

Hence, in view of assumption (vii), we have

$$
K\left(t_{2}, s\right)-K\left(t_{1}, s\right) \leqslant 0
$$

and the proof is complete. 
The next result indicates the utility of assumption (vi').

Theorem 10. Suppose that the function $K=K(t, s)$ satisfies assumptions (iv), (vi'), and (vii). Then $K$ satisfies assumption (vi).

Proof. Fix an arbitrary number $\varepsilon>0$. In view of assumption (iv) we deduce that there exists $\delta>0$ such that if $t_{1}, t_{2} \in \mathbb{R}_{+}$, $t_{1}<t_{2}$ and $t_{2}-t_{1}<\delta$ then

$$
\left|K\left(t_{2}, t_{1}\right)-K\left(t_{1}, t_{1}\right)\right| \leqslant \varepsilon .
$$

In the light of Lemma 9 the above inequality can be written equivalently in the form

$$
0 \leqslant K\left(t_{1}, t_{1}\right)-K\left(t_{2}, t_{1}\right) \leqslant \varepsilon
$$

Further, assume that $t_{1}, t_{2}$ are fixed. Take a partition $0=s_{0}<$ $s_{1}<\cdots<s_{n}=t_{1}$ of the interval $\left[0, t_{1}\right]$. Then, in view of assumptions $\left(\mathrm{vi}^{\prime}\right)$ and (vii) and Lemma 9, we obtain

$$
\begin{aligned}
& \sum_{i=1}^{n}\left|\left[K\left(t_{2}, s_{i}\right)-K\left(t_{1}, s_{i}\right)\right]-\left[K\left(t_{2}, s_{i-1}\right)-K\left(t_{1}, s_{i-1}\right)\right]\right| \\
& \quad=\sum_{i=1}^{n}\left\{\left[K\left(t_{2}, s_{i-1}\right)-K\left(t_{1}, s_{i-1}\right)\right]-\left[K\left(t_{2}, s_{i}\right)-K\left(t_{1}, s_{i}\right)\right]\right\} \\
& \quad=K\left(t_{1}, t_{1}\right)-K\left(t_{2}, t_{1}\right) .
\end{aligned}
$$

Hence we deduce that

$$
\bigvee_{s=0}^{t_{1}}\left[K\left(t_{2}, s\right)-K\left(t_{1}, s\right)\right]=K\left(t_{1}, t_{1}\right)-K\left(t_{2}, t_{1}\right)
$$

Finally, combining the above equality with (33), we complete the proof.

In what follows we show how the result contained in Theorem 5 can be applied to the Volterra-Wiener-Hopf integral equation (4). First of all let us recall that (4) is a special case of the Volterra-Stieltjes integral equation (6) if we put

$$
K(t, s)=\int_{0}^{s} k(t-z) d z
$$

for $(t, s) \in \Delta$. Obviously such a substitution has a sense under suitable assumptions concerning the function $k=k(u)$, which will be formulated later.

To adapt the assumptions of Theorem 5 to our situation let us observe that assumption (vii) is then automatically satisfied since $K(t, 0)=0$.

Let us observe that in order to ensure the well definiteness of the function $K=K(t, s)$ we have to assume that the function $k=k(u)$ is locally integrable over $\mathbb{R}_{+}$(in Lebesgue sense). Moreover, to adapt assumption (vi), let us notice that taking $t_{1}, t_{2} \in \mathbb{R}_{+}, t_{1}<t_{2}$, we have

$$
\begin{aligned}
\bigvee_{s=0}^{t_{1}} & {\left[K\left(t_{2}, s\right)-K\left(t_{1}, s\right)\right] } \\
& =\bigvee_{s=0}^{t_{1}}\left[\int_{0}^{s} k\left(t_{2}-z\right) d z-\int_{0}^{s} k\left(t_{1}-z\right) d z\right] \\
& =\bigvee_{s=0}^{t_{1}} \int_{0}^{s}\left[k\left(t_{2}-z\right)-k\left(t_{1}-z\right)\right] d z \\
& =\int_{0}^{t_{1}}\left[k\left(t_{2}-z\right)-k\left(t_{1}-z\right)\right] d z .
\end{aligned}
$$

In view of the above equality we can reformulate assumption (vi) in the following way.

$\left(\mathrm{vi}_{1}\right)$ For any $\varepsilon>0$ there exists $\delta>0$ such that for all $t_{1}, t_{2} \epsilon$ $\mathbb{R}_{+}$with $t_{1}<t_{2}, t_{2}-t_{1} \leqslant \delta$, the following inequality holds:

$$
\int_{0}^{t_{1}}\left[k\left(t_{2}-s\right)-k\left(t_{1}-s\right)\right] d s \leqslant \varepsilon
$$

In a similar way, assumption (viii) can be translated to the following form.

(viii $\left.{ }_{1}\right)$ The function $t \rightarrow \int_{0}^{t} k(t-s) d s$ is bounded on $\mathbb{R}_{+}$.

In order to present the last assumption in a more transparent form, let us substitute $u=t-s$ in the integral appearing in assumption ( viii $_{1}$ ). Then we get

$$
\int_{0}^{t} k(t-s) d s=\int_{0}^{t} k(u) d u
$$

Thus, the above condition concerning the local Lebesgue integrability of the function $k=k(u)$ in conjunction with the above observation implies that we should put the following assumption in place of ( viii $\left._{1}\right)$.

( viii $_{2}$ ) The function $k=k(u)$ is Lebesgue integrable over $\mathbb{R}_{+}$.

It is well-known that the Lebesgue integrability of the function $k=k(u)$ on the interval $\mathbb{R}_{+}$implies that the function

$$
t \rightarrow \int_{0}^{t} k(u) d u
$$

(the indefinite integral of $k$ ) is absolutely continuous on $\mathbb{R}_{+}$ (cf. $[13,17,18]$ ). This immediately implies that the function defined by (40) is uniformly continuous on $\mathbb{R}_{+}$.

Now, let us observe that the above formulated assumption $\left(\mathrm{vi}_{1}\right)$ connected with the Volterra-Wiener-Hopf integral equation (4) has rather inconvenient form and is not easy to verify in practice. Therefore, in our further investigations, we will utilize assumption ( $\left(\mathrm{vi}^{\prime}\right)$ instead of assumption (vi). Obviously, assumption ( $\mathrm{vi}^{\prime}$ ) will be adapted to the case of (4). 
To this end choose arbitrarily $t_{1}, t_{2} \in \mathbb{R}_{+}$with $t_{1}<t_{2}$. According to assumption ( $\left.\mathrm{vi}^{\prime}\right)$ the function

$$
s \longrightarrow K\left(t_{2}, s\right)-K\left(t_{1}, s\right)
$$

should be nonincreasing on the interval $\left[0, t_{1}\right]$. Taking into account that

$$
\begin{aligned}
K\left(t_{2}, s\right)-K\left(t_{1}, s\right) & =\int_{0}^{s} k\left(t_{2}-z\right) d z-\int_{0}^{s} k\left(t_{1}-z\right) d z \\
& =\int_{0}^{s}\left[k\left(t_{2}-z\right)-k\left(t_{1}-z\right)\right] d z,
\end{aligned}
$$

we have to impose the condition requiring that the function

$$
s \longrightarrow \int_{0}^{s}\left[k\left(t_{2}-z\right)-k\left(t_{1}-z\right)\right] d z
$$

is nonincreasing on the interval $\left[0, t_{1}\right]$.

Since the function (42) is absolutely continuous on the interval $\left[0, t_{1}\right]$, by the well-known facts from the theory of real functions [18], this requirement can be expressed equivalently in the following form:

$$
k\left(t_{2}-s\right)-k\left(t_{1}-s\right) \leqslant 0
$$

for $s \in\left[0, t_{1}\right]$. Obviously this means that the function $k=$ $k(u)$ is nonincreasing on $\mathbb{R}_{+}$.

On the other hand any monotone function is Riemann integrable. Thus, assuming additionally that $k: \mathbb{R}_{+} \rightarrow \mathbb{R}_{+}$we conclude that $k$ is nonincreasing and bounded on $\mathbb{R}_{+}$. It is known [13] that in this case the function

$$
t \longrightarrow \int_{0}^{t} k(u) d u
$$

is Lipschitz continuous on $\mathbb{R}_{+}$. In other words, in such a situation, we have that the function

$$
s \longrightarrow K(t, s)=\int_{0}^{s} k(t-z) d z
$$

is uniformly continuous on the interval $[0, t]$.

Keeping in mind the above conducted considerations we can formulate the following result concerning the VolterraWiener-Hopf integral equation (4).

Theorem 11. Assume that there are satisfied assumptions (i), (ii), and (iii) of Theorem 5. Moreover, we assume that the following conditions are satisfied.

(x) The function $k(u)=k: \mathbb{R}_{+} \rightarrow \mathbb{R}_{+}$is nonincreasing and integrable on $\mathbb{R}_{+}$.

(xi) There exists a positive solution $r_{0}$ of the inequality

$$
\|a\|+\left(\phi(r)+F_{1}\right) \bar{k} \leqslant r
$$

where

$$
\bar{k}=\int_{0}^{\infty}|k(u)| d u
$$

Then there exists at least one solution $x=x(t)$ of (4) in the space $B C\left(\mathbb{R}_{+}\right)$which has a limit at infinity.

Finally, let us mention that the result concerning the nonlinear integral equation (4) obtained in this section generalizes several ones which can be encountered in the literature (cf. $[5,19,20]$, e.g.).

\section{Further Discussions and Examples}

At the beginning of this section we intend to discuss some assumptions imposed on the terms of the integral equation of Volterra-Wiener-Hopf type (4) considered in the previous section.

Let us start with the requirement that the function $k=$ $k(u)$ transforms $\mathbb{R}_{+}$into itself and is nonincreasing on $\mathbb{R}_{+}$. Observe that in that case we allow the function $k$ to take negative values; that is, if we would assume that $k(u) \leqslant$ $k\left(u_{0}\right)<0$ for $u>u_{0}$, then we infer that $k$ is not integrable on $\mathbb{R}_{+}$and we obtain a contradiction with assumption (x).

Further on, let us notice that in our considerations connected with (6), assumption (vi') can be replaced by the following one.

$\left(\right.$ vi $\left.^{\prime \prime}\right)$ For arbitrary $t_{1}, t_{2} \in \mathbb{R}_{+}$such that $t_{1}<t_{2}$ the function $s \rightarrow K\left(t_{2}, s\right)-K\left(t_{1}, s\right)$ is nondecreasing on the interval $\left[0, t_{1}\right]$.

Indeed, in such a case, arguing similarly to the proof of Lemma 9 and Theorem 10, we can prove the following analogous results.

Lemma 12. Under assumptions ( $\left.v i^{\prime \prime}\right)$ and (vii), for arbitrarily fixed $s \in \mathbb{R}_{+}$, the function $t \rightarrow K(t, s)$ is nondecreasing on the interval $[s, \infty)$.

Theorem 13. Suppose that the function $K=K(t, s)$ satisfies assumptions (iv), (vi'), and (vii). Then $K$ satisfies assumption (vi).

Further, performing similar reasonings as at the end of Section 3, we can easily conclude that, in the case of VolterraWiener-Hopf equation (4), assumption ( $\left.\mathrm{vi}^{\prime \prime}\right)$ is equivalent to the requirement that the function $k=k(u)$ is nondecreasing on $\mathbb{R}_{+}$. This immediately yields that in order to ensure the integrability of the function $k$ over the interval $\mathbb{R}_{+}$we are forced to assume that $k: \mathbb{R}_{+} \rightarrow \mathbb{R}_{-}=(-\infty, 0]$.

Now, we are prepared to formulate other (nondecreasing) versions of Theorem 11 .

Theorem 14. Assume that there are satisfied assumptions (i), (ii), and (iii) of Theorem 5 and assumption (xi) of Theorem 11. Moreover, we assume that the following condition is satisfied.

$\left(\mathrm{x}^{\prime}\right)$ The function $k(u)=k: \mathbb{R}_{+} \rightarrow \mathbb{R}_{-}$is nondecreasing and integrable on $\mathbb{R}_{+}$.

Then there exists at least one solution $x=x(t)$ of (4) in the space $B C\left(\mathbb{R}_{+}\right)$which has a limit at infinity.

Obviously, the proof of Theorem 14 runs in a similar way as the proof of Theorem 11 . 
In what follows let us pay our attention to the case of Volterra-Wiener-Hopf integral equation (4) considered on a bounded interval $[0, T]$. This means that we consider the following integral equation of Volterra-Wiener-Hopf type:

$$
x(t)=a(t)+\int_{0}^{t} k(t-s) f(s, x(s)) d s
$$

for $t \in[0, T]$, where $T>0$ is a given number.

Observe that in this case we can replace assumption (i) by the assumption requiring that $a \in C[0, T]$ and we can delete assumption (iii). Similarly we can modify and adapt suitable assumptions (iv) and (vii). Summing up, we can formulate the following result concerning equation (49) for $t \in[0, T]$.

Theorem 15. Assume that the following hypotheses are satisfied:

(1) $a \in C[0, T]$;

(2) $f:[0, T] \times \mathbb{R} \rightarrow \mathbb{R}$ is continuous and there exists a function $\phi: \mathbb{R}_{+} \rightarrow \mathbb{R}_{+}$which is nondecreasing, $\phi(0)=$ $0, \lim _{t \rightarrow 0} \phi(t)=0$, and such that

$$
|f(t, x)-f(t, y)| \leqslant \phi(|x-y|)
$$

for all $t \in[0, T]$ and $x, y \in \mathbb{R}$;

(3) the function $k(u)=k:[0, T] \rightarrow \mathbb{R}$ is monotone on $[0, T]$;

(4) there exists a positive solution $r_{0}$ of the inequality

$$
\|a\|_{C[0, T]}+\left(\phi(r)+F_{1}\right) \bar{k} \leqslant r
$$

where $\bar{k}=\int_{0}^{T}|k(u)| d u$ and $F_{1}=\max \{|f(t, 0)|: t \in$ $[0, T]\}$.

Then there exists at least one solution $x=x(t)$ of (49) in the space $C[0, T]$.

Let us remark that the space $C[0, T]$ denotes the classical Banach space consisting of real functions being continuous on the interval $[0, T]$ and endowed by the classical maximum norm.

In the remainder of this section we provide a few examples associated with the Volterra-Wiener-Hopf integral equation (4) considered on the real half-axis $\mathbb{R}_{+}$. At the beginning we present examples of functions $k=k(u)$ satisfying requirement of Theorems 11 and 14 .

Example 1. Let us take the function $k$ having the form

$$
k(u)=\frac{1}{u^{2}+1} .
$$

Obviously $k: \mathbb{R}_{+} \rightarrow \mathbb{R}_{+}$and the function $k$ is nonincreasing on the interval $\mathbb{R}_{+}$. Moreover, the function $k$ is integrable over $\mathbb{R}_{+}$and

$$
\int_{0}^{t} k(u) d u=\arctan t
$$

Thus

$$
\bar{k}=\int_{0}^{\infty} k(u) d u=\frac{\pi}{2}
$$

Example 2. Consider the function $k(u)=e^{-u}$. Observe that this function satisfies assumption $(\mathrm{x})$ since it is decreasing and integrable on $\mathbb{R}_{+}$. Moreover, we have that

$$
\bar{k}=\int_{0}^{\infty} k(u) d u=\int_{0}^{\infty} e^{-u} d u=1,
$$

where $\bar{k}$ is the constant defined by (48).

Example 3. Now, consider the function $k=k(u)$ of the form

$$
k(u)=(u+1) e^{-u}
$$

It is easy to check that $k$ is decreasing and integrable on the interval $\mathbb{R}_{+}$. Moreover, we have that

$$
\bar{k}=\int_{0}^{\infty}(u+1) e^{-u} d u=2 .
$$

Example 4. Let us take into account the function $k$ defined by the formula

$$
k(u)=\frac{-1}{1+e^{u}} .
$$

Obviously, we can easily verify that $k: \mathbb{R}_{+} \rightarrow \mathbb{R}_{-}$and $k$ is increasing on $\mathbb{R}_{+}$. Moreover, we have

$$
\bar{k}=\int_{0}^{\infty} \frac{1}{1+e^{u}} d u=\ln 2 .
$$

In what follows we provide an example illustrating Theorem 11.

Example 5. Let us consider the Volterra-Wiener-Hopf integral equation having the form

$$
x(t)=\frac{t^{2}+1}{t^{2}+2}+\int_{0}^{t} \frac{1}{(t-s)^{2}+1} \sqrt[3]{x^{2}(s)+\arctan \left(\frac{s}{s^{2}+4}\right)} d s .
$$

Observe that (60) is a special case of (4) if we put

$$
\begin{aligned}
a(t) & =\frac{t^{2}+1}{t^{2}+2}, \\
k(u) & =\frac{1}{u^{2}+1},
\end{aligned}
$$

$$
f(t, x)=\sqrt[3]{x^{2}+\arctan \left(\frac{t}{t^{2}+4}\right)} .
$$

Let us verify that the terms involved in (60) satisfy the assumption of Theorem 11 .

Indeed, the function $a=a(u)$ satisfies assumption (i) and we have that $\|a\|=1$. Obviously, the function $f=f(t, x)$ defined by (63) is continuous on the set $\mathbb{R}_{+} \times \mathbb{R}$. To prove the second part of assumption (ii) we will use the following inequality:

$$
\left|\sqrt[3]{x^{2}+a}-\sqrt[3]{y^{2}+a}\right| \leqslant \sqrt[3]{(x-y)^{2}}
$$


(cf. [21]). Thus, in view of (64), we get

$$
\begin{aligned}
& |f(t, x)-f(t, y)| \\
& \quad \leqslant\left|\sqrt[3]{x^{2}+\arctan \left(\frac{t}{t^{2}+4}\right)}-\sqrt[3]{y^{2}+\arctan \left(\frac{t}{t^{2}+4}\right)}\right| \\
& \quad \leqslant \sqrt[3]{(x-y)^{2}} .
\end{aligned}
$$

Hence we infer that the function $f=f(t, x)$ satisfies assumption (ii) with the function $\phi(r)=r^{2 / 3}$. Obviously $\phi(0)=0, \phi$ is nondecreasing, and $\lim _{r \rightarrow 0} \phi(r)=0$.

In order to check that the function $f=f(t, x)$ satisfies assumption (iii) observe that

$$
f(t, 0)=\sqrt[3]{\arctan \left(\frac{t}{t^{2}+4}\right)} .
$$

Applying the standard methods of differential calculus, we obtain

$$
\begin{aligned}
F_{1}=\sup \{|f(t, 0)|: t \geqslant 0\} & =\sqrt[3]{\arctan \left(\frac{1}{4}\right)} \\
& =\sqrt[3]{0.2449 \ldots}=0.62564 \ldots .
\end{aligned}
$$

Next, in view of Example 1, we derive that the function $k$ given by (52) satisfies assumption (x) and $\bar{k}=\pi / 2$.

Finally, let us consider inequality (47) which now has the following form:

$$
1+\left(r^{2 / 3}+0.62564 \ldots\right) \frac{\pi}{2} \leqslant r .
$$

Using the standard methods of mathematical analysis we can show that there exists a number $\bar{r}$ belonging to the interval $(8,9)$ which satisfies the equation

$$
1+\left(r^{2 / 3}+0.62564 \ldots\right) \frac{\pi}{2}=r .
$$

Thus, this allows us to deduce that for any number $r_{0} \geqslant \bar{r}$ there is satisfied inequality (68). For example, we can accept that $r_{0}=9$.

Now, invoking Theorem 11, we infer that there exists at least one solution $x=x(t)$ of $(60)$ in the space $\mathrm{BC}\left(\mathbb{R}_{+}\right)$ which belongs to the ball $B_{9}$ and has a finite limit at infinity. Obviously the limit $\lim _{t \rightarrow \infty} x(t)$ belongs to the interval $[-9,9]$.

\section{Conflict of Interests}

The authors declare that there is no conflict of interests regarding the publication of this paper.

\section{References}

[1] S. Chandrasekhar, Radiative Transfer, Oxford University Press, London, UK, 1950.
[2] K. Deimling, Nonlinear Functional Analysis, Springer, Berlin, Germany, 1985.

[3] N. Dunford and J. T. Schwartz, Linear Operators, International Publishing, Leiden, The Netherlands, 1963.

[4] I. Podlubny, Fractional Differential Equations, vol. 198 of Mathematics in Science and Engineering, Academic Press, San Diego, Calif, USA, 1999.

[5] P. P. Zabrejko, A. I. Koshelev, M. A. Krasnosel'skii, S. G. Mikhlin, L. S. Rakovschik, and J. Stetsenko, Integral Equations, Nordhoff, Leyden, Mass, USA, 1975.

[6] E. Hopf, Mathematical Problems of Radiative Equilibria, Cambridge Tract 31, Cambridge University Press, Cambridge, UK, 1934.

[7] E. T. Copson, "On an integral equation arising in the theory of diffraction," The Quarterly Journal of Mathematics: Oxford: Second Series, vol. 17, pp. 19-34, 1946.

[8] J. F. Carlson and A. E. Heins, "The reflection of an electromagnetic plane wave by an infinite set of plates. I," Quarterly of Applied Mathematics, vol. 4, pp. 313-329, 1947.

[9] I. D. Abrahams, "On the application of the Wiener-Hopf technique to problems in dynamic elasticity," Wave Motion, vol. 36, no. 4, pp. 311-333, 2002.

[10] Y. A. Antipov, "Diffraction of a plane wave by a circular cone with an impedance boundary condition," SIAM Journal on Applied Mathematics, vol. 62, no. 4, pp. 1122-1152, 2002.

[11] J. Banaś, "Measures of noncompactness in the study of solutions of nonlinear differential and integral equations," Central European Journal of Mathematics, vol. 10, no. 6, pp. 2003-2011, 2012.

[12] J. Banaś and K. Goebel, Measures of Noncompactness in Banach Spaces, vol. 60 of Lecture Notes in Pure and Applied Mathematics, Marcel Dekker, New York, NY, USA, 1980.

[13] J. Appell, J. Banaś, and N. Merentes, Bounded Variation and Around, vol. 17 of De Gruyter Series in Nonlinear Analysis and Applications, Walter de Gruyter, Berlin, Germany, 2014.

[14] I. P. Natanson, Theory of Functions of Real Variable, Ungar, New York, NY, USA, 1960.

[15] J. Banaś and T. Zając, "A new approach to the theory of functional integral equations of fractional order," Journal of Mathematical Analysis and Applications, vol. 375, no. 2, pp. 375387, 2011.

[16] T. Zając, "Solvability of fractional integral equations on an unbounded interval through the theory of Volterra-Stieltjes integral equations," Zeitschrift für Analysis und ihre Anwendungen, vol. 33, no. 1, pp. 65-85, 2014.

[17] W. Rudin, Real and Complex Analysis, McGraw-Hill Book, New York, NY, USA, 3rd edition, 1987.

[18] R. Sikorski, Real Functions, PWN, Warsaw, Poland, 1958 (Polish).

[19] C. Corduneanu, Integral Equations and Applications, Cambridge University Press, Cambridge, UK, 1991.

[20] R. Stańczy, "Hammerstein equations with an integral over a noncompact domain," Annales Polonici Mathematici, vol. 69, no. 1, pp. 49-60, 1998.

[21] R. P. Agarwal, J. Banaś, K. Banaś, and D. O’Regan, “Solvability of a quadratic Hammerstein integral equation in the class of functions having limits at infinity," Journal of Integral Equations and Applications, vol. 23, no. 2, pp. 157-181, 2011. 


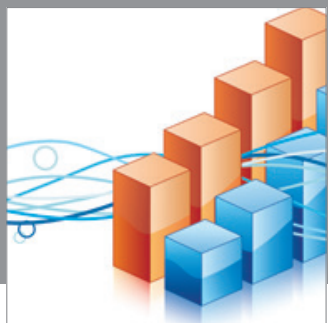

Advances in

Operations Research

mansans

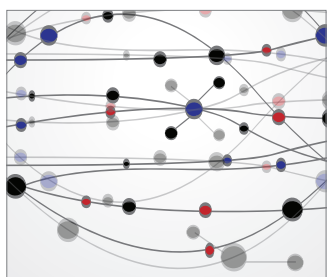

The Scientific World Journal
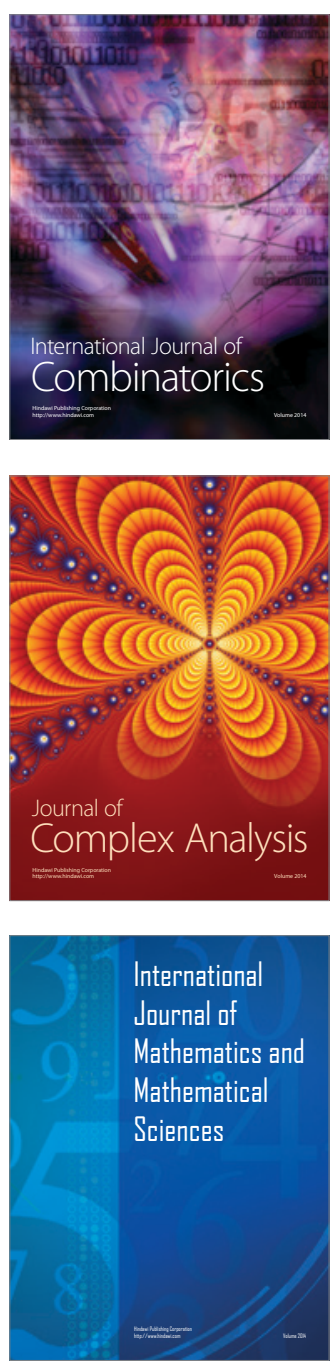
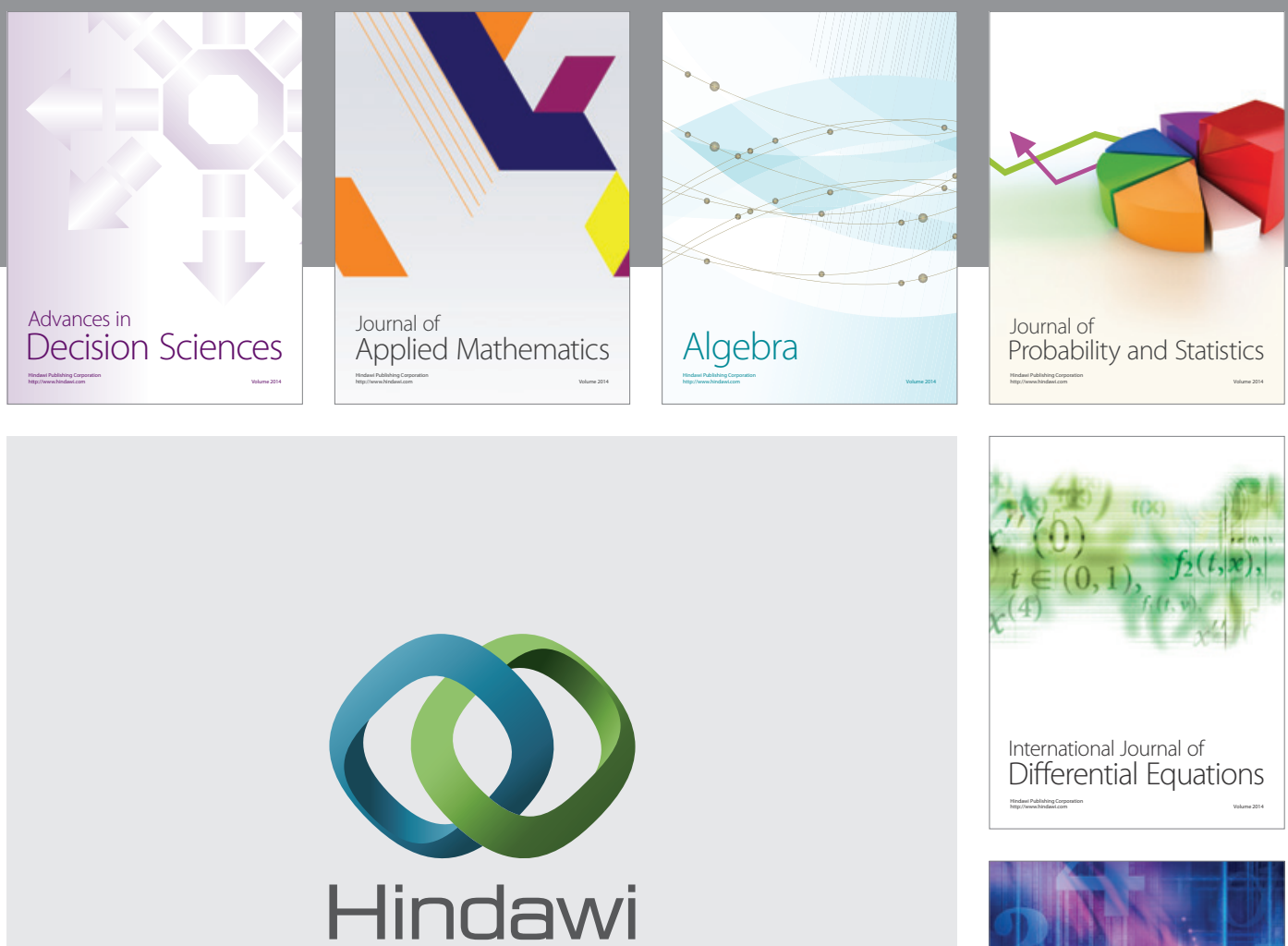

Submit your manuscripts at http://www.hindawi.com
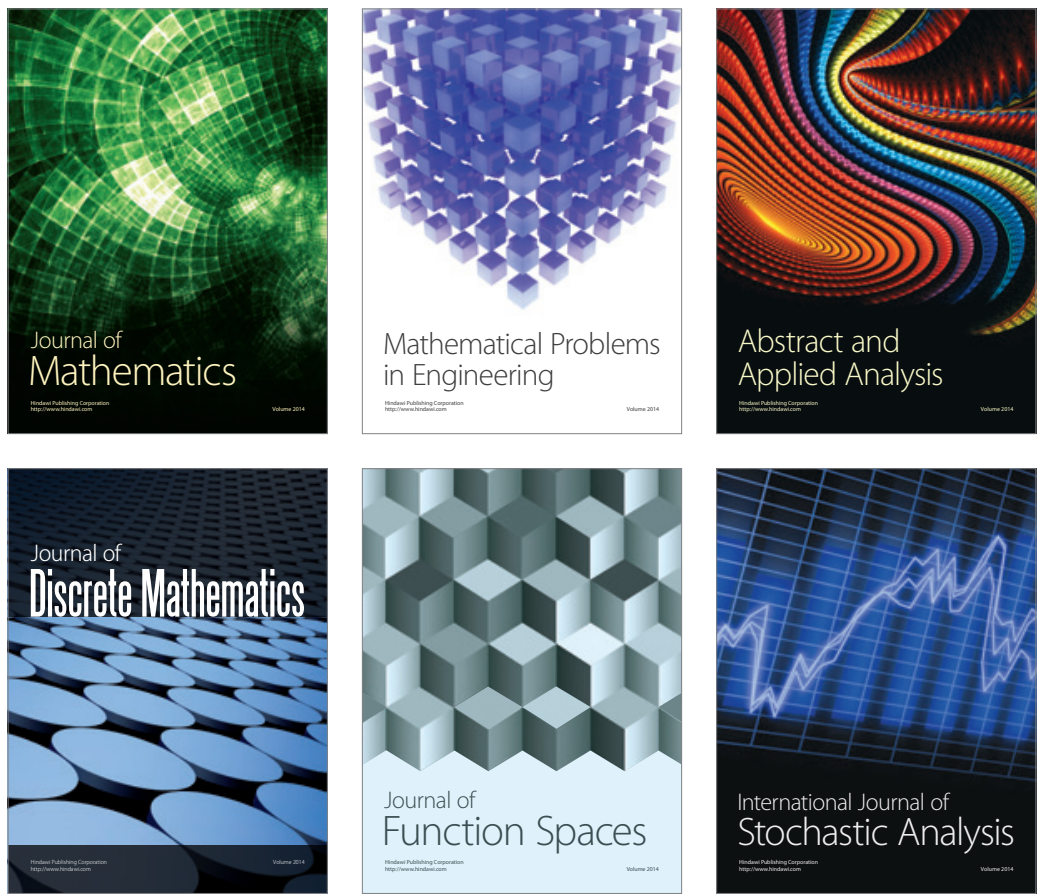

Journal of

Function Spaces

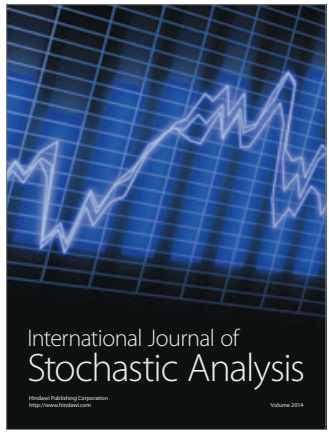

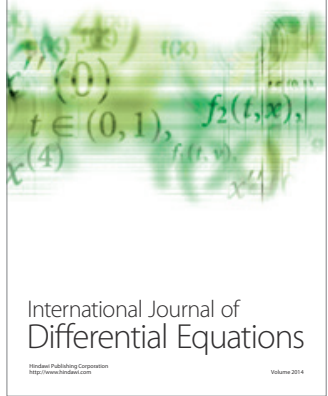
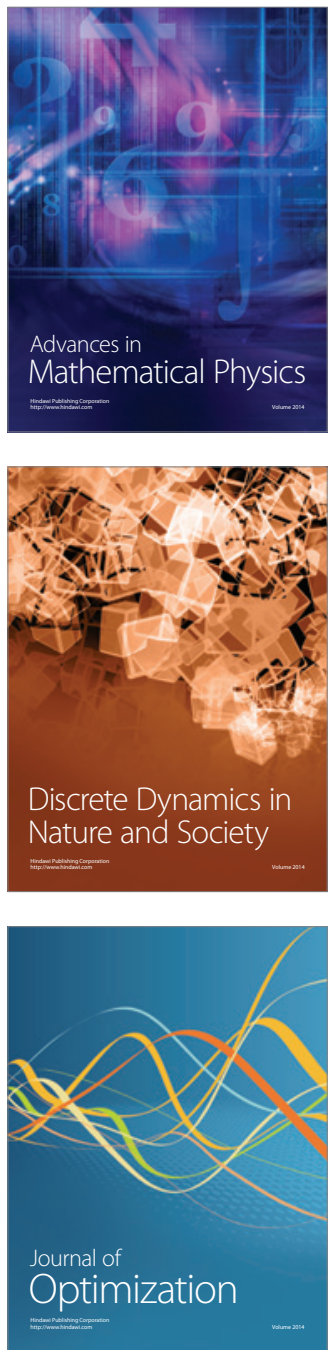